\title{
Molecular Structure of Gardenia Blue Pigments \\ by Reaction of Genipin with Benzylamine and Amino Acids
}

\begin{abstract}
Kaname Tsutsumiuchi ${ }^{1 *}$, Tomozou Toyoshima ${ }^{1}$, Fumiya Hasegawa ${ }^{1}$, Riku Terasawa ${ }^{1}$, Wataru Honda ${ }^{1}$, Minami Sakakibara ${ }^{1}$, Yasuyuki Ishida ${ }^{1}$, Yoshitomo Ikai $^{1}$, Ryo Ishibashi ${ }^{2}$, Kota Furuya ${ }^{2}$, Takashi Morimoto ${ }^{2}$, Kyoko Ishizuki $^{3}$, Yuzo Nishizaki ${ }^{3}$, Naoko Masumoto ${ }^{3}$, Naoki Sugimoto ${ }^{3}$, Kyoko Sato ${ }^{3}$, Hisao Oka ${ }^{4}$
\end{abstract}

${ }^{1}$ College of Bioscience and Biotechnology, Chubu University, 1200 Matsumoto, Kasugai, Aichi 487-8501, Japan

${ }^{2}$ San-Ei Gen F.F.I., Inc., 1-1-11 Miwa, Toyonaka, Osaka 561-8588, Japan

${ }^{3}$ National Institute of Health Sciences, 3-25-26 Tonomachi, Kawasaki, Kawasaki, Kanagawa 2109501, Japan

${ }^{4}$ Fuji Baking Group Research Laboratory, 1-50 Matsuzono, Mizuho, Nagoya 467-0065, Japan

\section{Supporting Information}

Table S1. Preparative LC conditions for compound B1 and B2

2

Table S2. LC-TOFMS conditions for compound B1 and B2 2

Figure S1. HPLC profiles of purified fractions, and mass and UV spectra of peak B1 and B2 $\cdots 3$ 
Table S1. Preparative LC conditions for intermediates B1 and B2

\begin{tabular}{|c|c|}
\hline LC syst em & $\begin{array}{l}\text { SHIMADZU: prominence LC(LC-20AT, SIL-20AC, CTO-20AC), PDA } \\
\text { (SPD-M20A), FRACTION COLLECTOR(FRC-10A) }\end{array}$ \\
\hline Column & At lant is Prep T3 $(10 \times 250 \mathrm{~mm}, 5 \mu \mathrm{m}$, Wat ers $)$ \\
\hline Column temp. & 400 \\
\hline Solvent & $\begin{array}{l}\text { A: } 0.1 \% \mathrm{HCOOH} / \mathrm{H}_{2} \mathrm{O} \text { B: } 0.1 \% \mathrm{HCOOH} / \mathrm{MeOH} \\
\text { isocratic: } \mathrm{B} 66 \%\end{array}$ \\
\hline Flow rate & $2.5 \mathrm{~mL} / \mathrm{min}$ \\
\hline PDA scan & $190-800 \mathrm{~nm}($ detect : $600 \mathrm{~nm})$ \\
\hline Injection volume & $200 \square 800 \mathrm{uL}$ \\
\hline
\end{tabular}

Table S2. LC-PDA-TOFMS conditions for intermediates B1 and B2

\begin{tabular}{|c|c|}
\hline LC M S system & $\begin{array}{l}\text { W aters UPLC:ACQUITY H-CLASS, PDA:ACQUIY e } \lambda \text {, } \\
\text { TOF-N S:XeVO-G } 20 \text { T of }\end{array}$ \\
\hline Coüm n & ACQU II Y UPLC BEH C $18(2.1 \times 50 \mathrm{~mm}, 1.7 \mu \mathrm{m}$, W aters $)$ \\
\hline Co um n tem p. & $40^{\circ} \mathrm{C}$ \\
\hline Solvent & $\begin{array}{l}\text { A: } 0.1 \% \mathrm{HCOOH} / \mathrm{H}_{2} \mathrm{O} \quad \mathrm{B}: 0.1 \% \mathrm{HCOOH} / \mathrm{NeOH} \\
\text { gradient: } \mathrm{B} 50 \%(0 \mathrm{~m} \mathrm{n}) \rightarrow 95 \%(9-11 \mathrm{~m} \mathrm{n})\end{array}$ \\
\hline F bw rate & $0.2 \mathrm{~mL} / \mathrm{m}$ in \\
\hline PDA scan & $190-800 \mathrm{~nm}$ \\
\hline Capillary voltage & $0.5 \sim 4.0 \mathrm{kV}$ \\
\hline Cone voltage & $10 \sim 100 \mathrm{~V}$ \\
\hline Source tem p. & $120^{\circ} \mathrm{C}$ \\
\hline Desolvation tem $p$ & $450^{\circ} \mathrm{C}$ \\
\hline Desolvation gas fow & $800 \mathrm{~L} \mathrm{hr}$ \\
\hline Cone gas fbw & $50 \mathrm{~L} / \mathrm{hr}$ \\
\hline bn mode & ES I (Reso ution m ode) \\
\hline MS scan & $m / 2100-4000$ \\
\hline
\end{tabular}




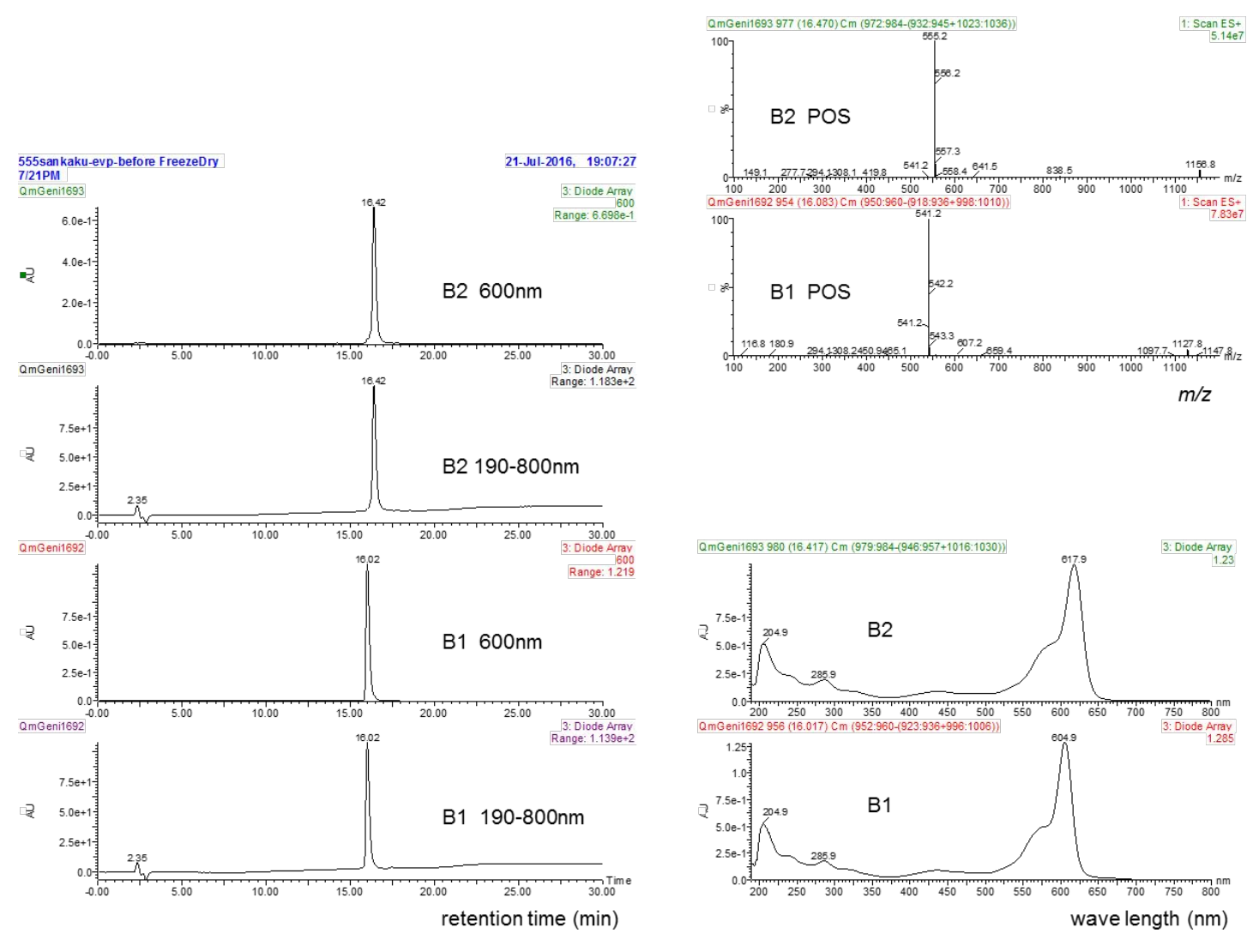

Figure S1. LC-PDA-TOFMS profiles of purified fractions, and mass and UV spectra of peak $\mathrm{B} 1$ and $\mathrm{B} 2$. 